\title{
ON THE USE OF MULTIPLE INSTANCE LEARNING FOR DATA CLASSIFICATION
}

\author{
${ }^{*}$ Nibras Z. Salih'
}

Walaa Khalaf ${ }^{1}$

1) Computer Engineering Department, College of Engineering, Mustansiriyah University, Baghdad, Iraq

\begin{abstract}
In the multiple instances learning framework, instances are arranged into bags, each bag contains several instances, the labels of each instance are not available but the label is available for each bag. Whilst in a single instance learning each instance is connected with the label that contains a single feature vector. This paper examines the distinction between these paradigms to see if it is appropriate, to cast the problem within a multiple instance framework. In single-instance learning, two datasets are applied (students' dataset and iris dataset) using Naïve Bayes Classifier (NBC), Multilayer perceptron (MLP), Support Vector Machine (SVM), and Sequential Minimal Optimization (SMO), while SimpleMI, MIWrapper, and MIBoost in multiple instances learning. Leave One Out Cross-Validation (LOOCV), five and ten folds Cross-Validation techniques $(5-\mathrm{CV}, 10-\mathrm{CV})$ are implemented to evaluate the classification results. A comparison of the result of these techniques is made, several algorithms are found to be more effective for classification in the multiple instances learning. The suitable algorithms for the students' dataset are MIBoost with MLP for LOOCV with an accuracy of $75 \%$, whereas SimpleMI with SMO for the iris dataset is the suitable algorithm for $10-\mathrm{CV}$ with an accuracy of $99.33 \%$.
\end{abstract}

Keywords: Multiple Instance Learning, Single Instance Learning, Classification algorithms, One Versus All (OVA) approach.

\section{Introduction}

Multiple Instance Learning (MIL) is a generalization of supervised learning, which is used to study a notion that correctly defines and propagates defined data on invisible data. In addition, MIL can be implemented to solve the problem of classification and regression, using a specific algorithm based on a series labeled instance. In single-instance learning, each object is connected with a label that contains a single feature vector, known as the traditional representation of machine learning. Whilst, in multiple instances learning, these features are known as instances, but the object known as the bag. The labels are connected to the bags that contain different instances of each bag by a separated feature vector. The description of data is the difference between single instance and multiple instances learning [1, 2, 3]. In the training phase, the data are divided into positive and negative bags. Therefore, the classifier is trained according to the bag's label. In most cases, binary classifications are involved with the MIL, where each instance has a label that assigns it to one of the two classes as positive or negative. Several instances consider a positive label although it is negative label, because there is an undefined label. Therefore, the advantage of MIL is to solve the label ambiguity, for instances within a bag. Whilst, the bag has a defined label $[4,5]$.

In the situations of missing label information, a MIL issue is sometimes viewed as a specific type of single-instance learning issue [5]. On the

*Corresponding Author: EGma020@uomustansiriyah.edu.iq 
other hand, the data in MIL may include insignificant or noisy features that make the preprocessing of these features important for performance improvement [6]. The prediction of drug activity is initially introduced in MIL by Dietterich et al. and according to the standard MIL, the hypothesis defined is that each positive bag contains at least one positive instance while all instances are negative in each negative bag [7]. Several algorithms that cover a variety of applications of MIL are suggested by Amores, Foulds, and Frank [3, 8].

MIL is considered in many applications such as medical applications [9], image restoration that depending on the content [10], education [11, 12], prediction of drug activities [7], and human action recognition [13].

Quellec et al. use MIL algorithms to identify anomalous regions and compare anomaly labels to the original label, using information source of mammography in computer-aided identification. Some algorithms are applied such as DD, miSVM, and MIBoost [9].

In [14], image sentiment classification is suggested. The technique is based on MIL, where the extracted blocks are separated into multiple scales, using two separate images to evaluate the model.

Xiao et al. introduce transfer learning to solve the MIL problem, when the bags are attached to various weak labels. Then, the bag's label is updated to improve the MIL by designing a repeating pattern [15].

Zoghlami et al. implement a MIL to overcome the problem of Ionizing Radiation Resistance in bacteria which focused on the relationships of instances between different bags and cross bag dependency [16]. Several studies explain the correlation between instances within a bag in MIL, and compared it with single instance learning. Alpaydin et al. compare multiple instances with single instance classification, to identify the problem for both paradigms. The instance level with the noise is combined and an SVM is used as the main classifier. A variety of synthetic MIL tasks at different complexities are described in [17]. Divya Tomar and Sonali Agarwal consider that each bag containing a different value from the other bag using MIL. A twin SVM is applied and compared to other classifiers in [18]. Carbonneau et al. survey the characteristics and application of MIL, by dividing them into categories. The tasks of MIL algorithms and their influence to identify problem characteristics, for classification tasks are described in [19].

To solve the MIL problem, MIWrapper, SimpleMI, and MIBoost are implemented for some related works. Zafra et al. present MIL to predict student failure, using a single instance and MIL. The authors utilize several algorithms in MIL including SimpleMI, MIWrapper, and MIBoost, and compare the result of single instance and MIL [5]. Kumar et al. predict students' results in the mid-course. The accuracy rate and consumed time are considered to build the model performance, of single instance and MIL algorithms. Two algorithms are applied using decision stump in a single instance. While, SimpleMI in MIL, with the conclusion that MIL is better than single instance learning [12].

Vanwinckelen et al. [3] present a correlation between instance level and bag level of the set classifiers by analyzing the results. This correlation is relatively weak for both accuracy and area under the curve. They conclude that better classifiers for bags are not necessarily good for instances, since this depends on some particular issues. Some algorithms are applied in MIL such as MISMO, MIBoost, SimpleMI, and 
MIWrapper. Taser et al. [2] present an ensemble learning that depends on the MIL approach, to enhance the classification efficiency. Taser et al applied a group of algorithms that depends on the MIL technique including MIWrapper and SimpleMI that correspond to classical algorithms like C4.5, Naive Bayes, MLP, and SVM. Thereafter, AdaBoost and Bagging algorithms are implemented on the same datasets.

This paper introduces a new representation in the active educational environment, based on two learning frameworks (single instance learning and MIL). The students' dataset and iris plant dataset are applied to the single instance learning. The goal of this paper is to transform these datasets into MIL, with comprehensive analysis to improve the performance of single instance learning. Several algorithms are applied to the single instance including, SVM, Naïve Bayes, and MLP, while MIWrapper, SimpleMI, and MIBoost are implemented in the MIL. Three techniques are used to test the datasets, which are Leave one out Cross-Validation, five, and ten folds Cross-Validation. Seven metrics are applied, which are sensitivity, specificity, precision, F-measure, ROC, PRC Area, and accuracy. Therefore, the main contribution of this paper includes a brief study on the integration of single-instance learning and MIL in machine learning. This paper aims to improve the classification results in single instance learning using MIL, and to enable educators to understand the student's performance and predict their instructional activities.

The paper is divided into the following sections, the experiment design is described in Section 2, material and approaches are presented in Section 3, the results and discussion are explained in Section 4, while the conclusion is presented in Section 5.

\section{Experiment Design}

Weka is a data mining tool designed by Waikato University. The tool depends on the JAVA language, which includes several algorithms of the machine learning processes.

Weka is an open-source software applying different techniques as data preprocessing, regression, and clustering. Weka usually uses the data file with ARFF extension (Attribute Relation File Format). There are two different parts: the first part is the header information which includes the relation name, the name, and type of the attributes, while the second part contains the data that involve the values of these attributes [2, 5, 20]. Figure 1.a shows a single instance learning, where each object has a single feature vector. Figure 1.b shows the MIL, where each object has multiple feature vectors (four instances) separated by "In" so that the appropriate information is described below.

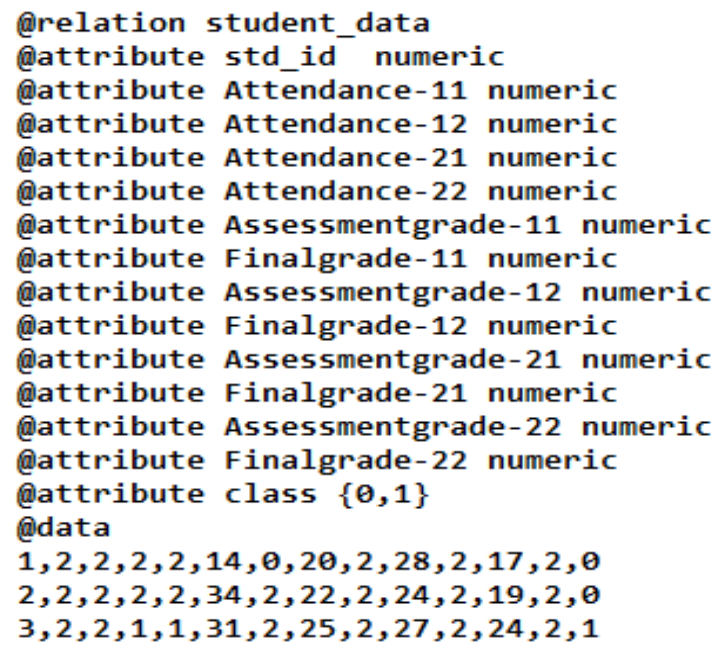

a. Single instance learning representation. 


\section{Qrelation Student_data-bag

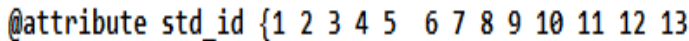 \\ 14151617181920212223242526272829 \\ $30313233343536373839404142 \quad 4344\}$ \\ @attribute bag relational \\ Qattribute Attendance numeric \\ @attribute Assessmentgrade numeric \\ @attribute Finalgrade numeric \\ Qend bag \\ Qattribute class $\{0,1\}$ \\ @data \\ $1, " 2,14,0 \backslash \mathrm{n} 2,20,2 \backslash \mathrm{n} 2,28,2 \backslash \mathrm{n} 2,17,2 ", 0$ \\ 2 ,"2,34,2\n2,22,2\n2,24,2\n2,19,2",0 \\ $3, " 2,31,2 \backslash n 2,25,2 \backslash n 1,27,2 \backslash n 1,24,2 ", 1$ \\ b. Multiple instance learning representation.}

Figure 1. Exemplification of students' performance data with WEKA format.

The confusion matrix is constructed by depending on the four-parameter of binary classification, which are True Positive (TP), True Negative (TN), False Positive (FP), and False Negative (FN). Seven metrics are applied to evaluate the classification performance including, sensitivity, specificity, precision, Fmeasure, ROC, PRC Area, and accuracy. These metrics are defined based on the various equations to the identification problem.

$$
\begin{aligned}
& \text { Precision }=\frac{\mathrm{TP}}{\mathrm{TP}+\mathrm{FP}} \\
& \text { Recall }=\frac{\mathrm{TP}}{\mathrm{TP}+\mathrm{FN}} \\
& \text { Specificity }=\frac{\mathrm{TN}}{\mathrm{TN}+\mathrm{FP}} \\
& \text { F-measure }=2 \times \frac{\text { Precision } \times \text { Recall }}{\text { Precision }+ \text { Recall }} \\
& \text { Accuracy }=\frac{\mathrm{TP}+\mathrm{TN}}{\mathrm{TP}+\mathrm{FP}+\mathrm{TN}+\mathrm{FN}} * 100 \%
\end{aligned}
$$

The ROC is the receiver operating characteristic curve, which is used to understand the performance of the classifier. ROC curve is sensitive for a balanced dataset that depends on two dimensions false positive rate and true positive rate.
Whilst the Precision-Recall Curve (PRC) is very sensitive when an unbalanced or noisy dataset is considered, it has two dimensions, the recall on the $\mathrm{x}$-axis and the precision on the $\mathrm{y}$-axis. ROC and PRC are used as one measure to compare the performance model at various threshold settings [21, 22].

\subsection{Data Description}

Two datasets are considered here, actual and classical (standard) datasets. In the first phase, the actual data are collected from the Mustansiriyah University of Iraq for three academic years. The relationship between main subjects, calculus, mathematical analysis, and control systems is suggested. This relationship shows how the student's success in calculus and mathematical analysis influences his or her ability to join a control system subject. The dataset consists of two courses for each stage, in the first stage calculus I and calculus II. Then, in the second stage, the mathematical analysis I and mathematical analysis II, to predict the control system subject at the third stage. Moreover, it involves forty-four instances, twelve attributes, and two classes which are pass or fail. The attribute information about calculus and mathematical analysis subjects are the following:

1. Attendance has three values $[0,1,2]$, the last alarm is indicated by a 0 , the first alarm is indicated by 1 , and 2 denotes that no absence was already observed.

2. Assessment grade has forty values [0 40], involves three activities: quiz modules, the test of the mid-semester, and students' assignments.

3. The final grade has three values $[0,1,2]$. The number 0 means that the student failed in both the first and the second attempts. The number 1 means that the student failed on the first attempt but 
succeeded on the second attempt. Number

2 denotes that the student passed the test from the first attempt.

The final information is the class that is related to the student's success or failure in the control systems subject where 0 (denotes the student's success) or 1 (denotes the student's failure). Figure 2.a illustrates the information about each student in single instance learning. Figure 2.b shows the bag detail in MIL.

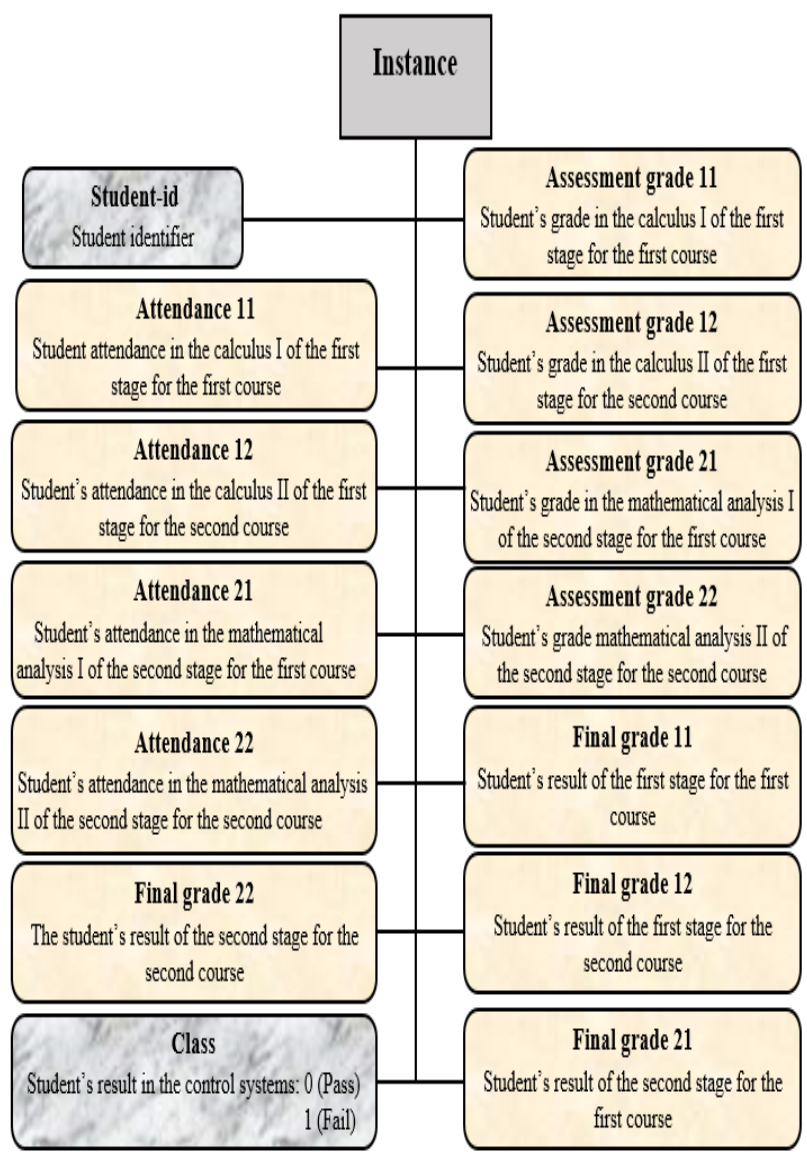

a. Student's information in single instance learning.

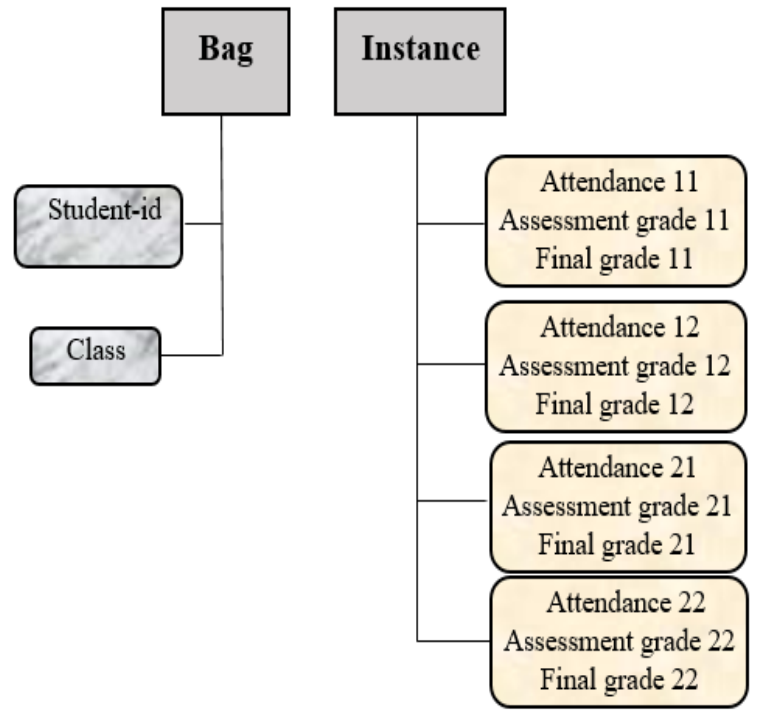

b. Student's information in MIL.

Figure 2. Student's information.

In the second phase, the iris dataset (classical) is utilized to assess the efforts. It consists of 150 instances, four attributes (sepal length, sepal width, petal length, and petal width), and three classes which are Setosa, Versicolor, and Virginica, each class has fifty instances. The first class can be linearly separated from other classes but the Versicolor and Virginica can't be separated linearly [23].

\section{Material and Approaches}

Single instance learning is the traditional supervised learning that consists of single feature vectors with a label. Whilst MIL contains instances by a separate feature vector, no detail about instances' label within a bag but the bag has a detailed label. The MIL described as follows, let $\mathrm{R}$ the space of the instance, $J$ the dataset consists of a group of bags that denotes to $J=\left\{\left(b_{1}, l_{1}\right),\left(b_{2}, l_{2}\right),\left(b_{3}, l_{3}\right), \ldots,\left(b_{m}, l_{m}\right)\right\}$.

Where $m$ is the total of training bags, $b$ is the bag and $l$ is the label.

Each bag contains a set of instance that denotes $b_{i}=\left\{x_{i 1}, x_{i 2}, x_{i 3}, \ldots, x_{i n}\right\} \subset R$, where $n$ is the number of instances of each bag. The objective of MIL is to label hidden bags 
correctly and to solve the classification problem. However, in a single instance learning each bag involves just one instance where $b_{i}=\left\{x_{n 1}\right\}$ for the dataset $[2,24]$.

Figure 3 shows the difference between single instance and MIL learning.
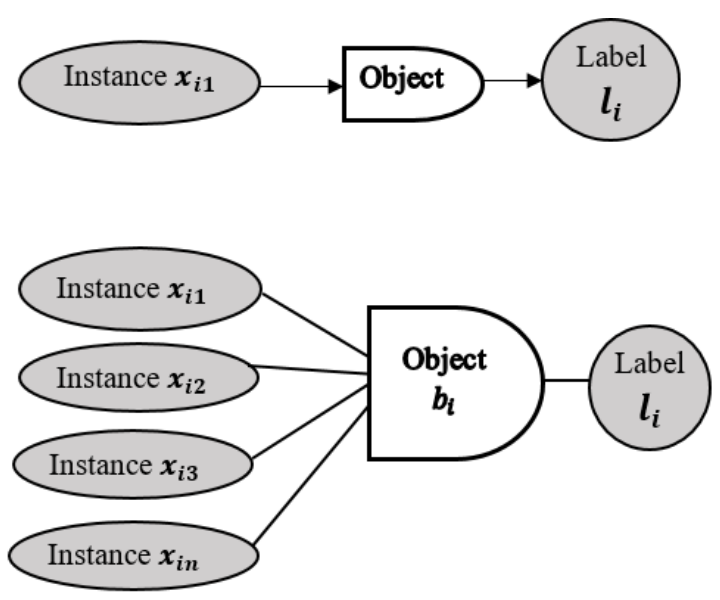

Figure 3. Single instance and multiple instance learning.

SimpleMI is an easy and quick algorithm introduced by Lin Dong which solves multiple instance classification problems, using singleinstance learning. The objective of this approach is to generate a single instance, to depict the total bag. In Weka, three methods applied in SimpleMI are arithmetic average, geometric average, and combined feature of a bag. The arithmetic average is implemented as follows:

$A(X)=\frac{1}{|X|} \sum_{x \in X}|(x)|$

Where $x$ is the total instances of each bag, $X$ is the number of instances and $A(X)$ is the mean of instance [25].

The Wrapper method is suggested by Xin $\mathrm{Xu}$ and Eibe Frank, it becomes one of the most straightforward algorithms, depending on the collective assumption, that all instances produce equal and separate contributions to the label of the bags. Weight value configured for each instance inside the bag, the instance gets the same weight value. The benefit of this method is to produce a class probability prediction that deals with weights of instance [26].

In boosting algorithms, several weak classifiers are created through the re-weighting of training instances. A robust classifier can be produced by merging these weak classifiers. AdaBoost is already updated into multiple instance learners named MIBoost, that also introduced by Xin $\mathrm{Xu}$ and Eibe Frank. MIBoost aims to provide a classifier to the model for each iteration also known as ensemble learning $[9,26]$.

\subsection{Multiclass Classification Problem}

In the classical dataset (iris plant dataset), OneVersus-All (OVA) approach is applied for a multiclass classification problem.

OVA approach uses three classifiers, to solve the three-class classification problem (Setosa, Versicolor, and Virginia).

Therefore every classifier takes one class to be positive, whereas the rest class is combined into the negative. In OVA the $k$ classifiers are equal to the class number. During the validation phase, each of the binary classifiers sends a pattern to generate the positive output that denotes the output class.

When the positive result is not an individual output, some method is used to break the correlation, that gives the confidence to the classifiers to choose the final result, and to predict the highest performance. Score vector is used in OVA. Let $V=\left\{v_{1}, v_{2}, \ldots, v_{i}\right\}$, where $V$ is the score vector and $v_{i} \in[0,1]$ that corresponds to class $i$ to assess the output values $[27,28]$. To convert the iris dataset from single instance learning into MIL. Three data files are written by ARFF extensions. First, Setosa class against Versicolor and Virginica classes. Second, the Versicolor class against Setosa and 
Virginica classes, and finally, Virginica class against Setosa and Versicolor classes.

\section{Results and Analysis}

Two different paradigms are applied on two datasets, which are single instance learning and MIL. In the first test, the NBC, MLP, and SVM are performed on the student dataset. In addition, the SMO with the poly kernel as SVM classifier, and the same algorithms mentioned above are performed on the iris dataset, using single instance learning. In the next test, MIWrapper, SimpleMI, MIBoost are applied to student and iris datasets using MIL. Then, different techniques are used to evaluate the performance of the classifier, LOOCV, and 5$\mathrm{CV}$ for five times, $10-\mathrm{CV}$. The results of these techniques are compared against each other for both paradigms. To understand the performance of each classifier, the results of the student dataset of the above-mentioned metrics for the LOOCV and 5-CV are shown in Table 1.

MIBoost with MLP is outperforming in terms of Sensitivity, Precision, F-measure, ROC, PRC Area, and Accuracy for LOOCV.

MIWrapper with NBC is outperforming in terms of Sensitivity, ROC, and Accuracy for 5-CV. The suitable results of this experiment have been underlined, SimpleMI with SMO is the best classifier in the MIL, when the iris dataset is applied.
Therefore, the result of SimpleMI, MIWrapper, and MIBoost classifiers becomes a higher average classification for most metrics than single instance learning of LOOCV, 5-CV, and $10-\mathrm{CV}$ when the MIL is applied. In singleinstance learning, the student dataset represents twelve dimensions features, while in MIL, the student dataset reduces to three dimensions features as shown in Figure 1.

Moreover, the iris dataset represents four dimensions features in single instance learning but the dimensions features are reduced in MIL to two dimensions features. The reduced dimension features in the MIL are the main reason for improving the classification of results.

Table 2 shows the results of the iris dataset for different classifiers (NBC, MLP, and SMO) using 10-CV. The ROC and PRC are utilized like a single metric. Therefore, the ROC and PRC values became higher when MIL is applied to the dataset for several algorithms, this proves the effective results for classifiers. 
Table 1. The classification results for students' dataset of the single instance and MIL.

\begin{tabular}{|c|c|c|c|c|c|c|c|c|}
\hline & & & & LOOCV & & & & \\
\hline \multirow{4}{*}{ 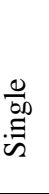 } & Metrics & Sensitivity & Specificity & Precision & F-Measure & ROC & PRC Area & Accuracy \\
\hline & NBC & 0.659 & 0.740 & 0.656 & 0.657 & 0.590 & 0.610 & $65.90 \%$ \\
\hline & MLP & 0.636 & 0.703 & 0.636 & 0.636 & 0.643 & 0.679 & $63.63 \%$ \\
\hline & SVM & 0.659 & 0.777 & 0.650 & 0.652 & 0.624 & 0.601 & $65.90 \%$ \\
\hline \multirow{7}{*}{ 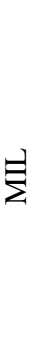 } & SimpleMI. NBC & 0.682 & 0.777 & 0.676 & 0.678 & 0.680 & 0.681 & $68.1818 \%$ \\
\hline & SimpleMI. MLP & 0.705 & 0.814 & 0.698 & 0.698 & 0.638 & 0.624 & $70.4545 \%$ \\
\hline & MIWrapper. NBC & 0.682 & 0.851 & 0.674 & 0.664 & 0.758 & 0.762 & $68.18 \%$ \\
\hline & MIWrapper. MLP & 0.682 & $\underline{0.925}$ & 0.691 & 0.640 & 0.717 & 0.745 & $68.18 \%$ \\
\hline & MIWrapper. SVM & 0.659 & 0.777 & 0.650 & 0.652 & 0.679 & 0.661 & $65.909 \%$ \\
\hline & MIBoost. NBC & 0.727 & 0.777 & 0.727 & 0.727 & 0.739 & 0.745 & $72.72 \%$ \\
\hline & MIBoost. MLP & $\underline{0.750}$ & 0.814 & $\underline{0.748}$ & $\underline{0.748}$ & $\underline{0.808}$ & $\underline{0.830}$ & $75 \%$ \\
\hline
\end{tabular}

$($ Mean \pm Std) for 5-CV

\begin{tabular}{|c|c|c|c|c|c|c|c|c|}
\hline \multirow{3}{*}{$\begin{array}{l}\frac{0}{0 D} \\
\Xi \approx\end{array}$} & NBC & $0.636 \pm 0.022$ & $0.695 \pm 0.03$ & $0.638 \pm 0.02$ & $0.637 \pm 0.021$ & $0.603 \pm 0.023$ & $0.617 \pm 0.015$ & $63.636 \pm 2.27$ \\
\hline & MLP & $0.672 \pm 0.012$ & $0.769 \pm 0.016$ & $0.666 \pm 0.015$ & $0.668 \pm 0.015$ & $0.677 \pm 0.01$ & $0.705 \pm 0.017$ & $67.27 \pm 1.24$ \\
\hline & SVM & $0.636 \pm 0.032$ & $0.74 \pm 0.074$ & $0.6312 \pm 0.02$ & $0.629 \pm 0.031$ & $0.606 \pm 0.03$ & $0.587 \pm 0.02$ & $63.636 \pm 3.21$ \\
\hline \multirow{7}{*}{$\exists$} & SimpleMI. NBC & $0.686 \pm 0.04$ & $0.754 \pm 0.067$ & $0.686 \pm 0.039$ & $0.684 \pm 0.041$ & $0.699 \pm 0.031$ & $0.706 \pm 0.029$ & $68.63 \pm 4.37$ \\
\hline & SimpleMI. MLP & $0.663 \pm 0.043$ & $0.754 \pm 0.056$ & $0.659 \pm 0.043$ & $0.659 \pm 0.044$ & $0.648 \pm 0.063$ & $0.648 \pm 0.048$ & $66.363 \pm 4.37$ \\
\hline & MIWrapper. NBC & $\underline{0.718 \pm 0.02}$ & $0.88 \pm 0.03$ & $0.718 \pm 0.025$ & $0.701 \pm 0.021$ & $\underline{0.777 \pm 0.046}$ & $0.786 \pm 0.056$ & $\underline{71.81 \pm 2.032}$ \\
\hline & MIWrapper. MLP & $0.709 \pm 0.037$ & $\underline{0.962 \pm 0.026}$ & $\underline{0.745 \pm 0.053}$ & $0.665 \pm 0.045$ & $0.751 \pm 0.019$ & $0.778 \pm 0.017$ & $70.90 \pm 3.734$ \\
\hline & MIWrapper. SVM & $0.672 \pm 0.026$ & $0.754 \pm 0.061$ & $0.672 \pm 0.03$ & $0.668 \pm 0.026$ & $0.663 \pm 0.051$ & $0.658 \pm 0.038$ & $67.27 \pm 2.59$ \\
\hline & MIBoost. NBC & $0.7 \pm 0.029$ & $0.762 \pm 0.061$ & $0.701 \pm 0.027$ & $0.698 \pm 0.028$ & $0.772 \pm 0.022$ & $\underline{0.793 \pm 0.023}$ & $70 \pm 2.9632$ \\
\hline & MIBoost. MLP & $0.718 \pm 0.03$ & $0.821 \pm 0.048$ & $0.715 \pm 0.032$ & $\underline{0.712 \pm 0.03}$ & $0.765 \pm 0.0290$ & $0.779 \pm 0.02$ & $71.81 \pm 3.049$ \\
\hline
\end{tabular}

Table 2. The classification results for the iris dataset of the single instance learning and MIL.

\begin{tabular}{|c|c|c|c|c|c|c|c|c|c|c|}
\hline \multirow{3}{*}{ Metrics } & \multirow{2}{*}{\multicolumn{3}{|c|}{$\begin{array}{c}\text { Single instance learning } \\
10-C V\end{array}$}} & \multicolumn{7}{|c|}{ MIL 10-CV } \\
\hline & & & & \multicolumn{3}{|c|}{ SimpleMI } & \multicolumn{2}{|c|}{ MIWrapper } & \multicolumn{2}{|c|}{ MIBoost } \\
\hline & NBC & MLP & $\begin{array}{c}\text { SMO } \\
\text { poly kernel }\end{array}$ & NBC & MLP & $\begin{array}{c}\text { SMO } \\
\text { poly kernel }\end{array}$ & NBC & MLP & NBC & MLP \\
\hline Sensitivity & 0.967 & 0.973 & 0.973 & 0.978 & $\underline{0.989}$ & $\underline{0.989}$ & 0.978 & 0.906 & 0.969 & 0.98 \\
\hline Specificity & 0.983 & 0.986 & 0.986 & 0.986 & $\underline{0.993}$ & 0.986 & 0.99 & 0.96 & 0.976 & 0.986 \\
\hline Precision & 0.967 & 0.973 & 0.974 & 0.978 & $\underline{0.989}$ & $\underline{0.989}$ & 0.978 & 0.905 & 0.969 & 0.98 \\
\hline F-Measure & 0.967 & 0.973 & 0.973 & 0.978 & $\underline{0.989}$ & $\underline{0.989}$ & 0.977 & 0.90 & 0.969 & 0.977 \\
\hline $\mathrm{ROC}$ & 0.992 & $\underline{0.998}$ & 0.982 & 0.968 & $\underline{0.998}$ & 0.99 & 0.978 & 0.945 & 0.992 & 0.988 \\
\hline PRC Area & 0.985 & 0.995 & 0.958 & 0.959 & $\underline{0.998}$ & 0.984 & 0.98 & 0.952 & 0.992 & 0.987 \\
\hline \multirow[t]{2}{*}{ Accuracy } & 96.667 & 97.3333 & 97.333 & 96.666 & 98 & $\underline{99.333}$ & 95.333 & 80 & 95.333 & 96 \\
\hline & $\%$ & $\%$ & $\%$ & $\%$ & $\%$ & $\%$ & $\%$ & $\%$ & $\%$ & $\%$ \\
\hline
\end{tabular}




\section{Conclusion}

MIL has a great interest in many societies rather than single instance learning. In this paper MIL is presented with single instance learning, to see if it is appropriate to cast the problem within MIL. These paradigms are proposed to solve the classification problem of the educational environment with the assistance of NBC, MLP, and SVM in single instance learning.

Then, MIWrapper, SimpleMI, and MIBoost are presented in the MIL that corresponds to the algorithms of single instance learning. The proposed approach is applied on two datasets which are the students' dataset (actual dataset) and the iris dataset (classical dataset), it is converted into MIL with extensive analysis, to improve the performance of single instance learning.

The studies show that student marks for one subject in academic courses, of the first and second year can be reliably predicted when MIL is applied. The results show that the best algorithms are the MIBoost with MLP for the students' dataset whereas SimpleMI with SMO for the iris dataset.

The future work is to expand the students' dataset to increase the prediction's reliability.

The other algorithms of MIL which are not applied in this paper such as two-level classification can be applied to the students' dataset.

\section{Acknowledgments}

My sincere appreciation and thanks to the University of Mustansiriyah for the guidance and support. Also, all thanks and appreciation to those who helped me and gave me scientific advice in this research.

\section{Conflict of interest}

The publication of this article causes no conflict of interest.

\section{References}

1. Melki, G., Cano, A. and Ventura, S. (2018). "MIRSVM: multi-instance support vector machine with bag representatives". Pattern Recognition, Vol. 79, pp. 228-241.

2. Taşer, P. Y., Birant, K. U. and Birant, D. (July 2019). "Comparison of ensemblebased multiple instances learning approaches", in 2019 IEEE International Symposium on Innovations in Intelligent Systems and Applications, pp. 1-5.

3. Vanwinckelen, G., Fierens, D. and Blockeel, H. (2016). "Instance-level accuracy versus bag-level accuracy in multi-instance learning". Data mining and knowledge discovery, Vol. 30, No. 2, pp. 313-341.

4. Xiao, Y., Yang, X. and Liu, B. (2020). “A new self-paced method for multiple instances boosting learning". Information Sciences, Vol. 515, pp. 80-90.

5. Zafra, A., Romero, C. and Ventura, S. (2011). "Multiple instances learning for classifying students in learning management systems". Expert Systems with Applications, Vol. 38, No. 12, pp. 15020-15031.

6. Chai, J., Chen, B., Liu, F., Chen, Z. and Ding, X. (2017). "Multiple-Instance feature extraction at the bag and instance levels using the maximum trace-difference criterion". Information Sciences, Vol. 385, pp. 353-377.

7. Dietterich, T. G. Lathrop, R.H., LozanoPerez, T. (1997). "Solving the multiple instance problem with axis-parallel rectangles". Artif. Intell, Vol. 89, No. 1, pp. 31-71.

8. Amores, J. (2013). "Multiple instance classification: Review, taxonomy, and comparative study". Artificial intelligence, Vol. 201, pp. 81-105, 
9. Quellec, G., Lamard, M., Cozic, M., Coatrieux, G. and Cazuguel, G. (2016). "Multiple-instance learning for anomaly detection in digital mammography". IEEE transactions on medical imaging, Vol. 35, No. 7, pp. 1604-1614.

10. Xu, Y. Y. (2016). "Multiple-instance learning-based decision neural networks for image retrieval and classification". Neurocomputing, Vol. 171, pp. 826-836.

11. Herrera, F., Ventura, S., Bello, R., Cornelis, C., Zafra, A., Sánchez-Tarragó, D. and Vluymans, S. (2016). "Multiple instance learning”, in Multiple instance learning, Springer, Cham, pp. 17-33.

12. Kumar, S. A., and Vijayalakshmi, M. N. (2018). "Efficiency of Multi-instance Learning in Educational Data Mining", in Knowledge Computing and Its Applications, Springer, Singapore, pp. 4764.

13. Yi, Y. and Lin, M. (2016). "Human action recognition with graph-based multipleinstance learning". Pattern Recognition, Vol. 53, pp.148-162.

14. Rao, T., Xu, M., Liu, H., Wang, J., et al. (September 2016). "Multi-scale blocks based image emotion classification using multiple instance learning", in 2016 IEEE International Conference on Image Processing (ICIP), pp. 634-638.

15. Xiao, Y., Liang, F. and Liu, B. (2020) "Transfer Learning-Based Multi-Instance Learning Method with Weak Labels". IEEE transactions on cybernetics.

16. Zoghlami, M., Aridhi, S., Maddouri, M., and Nguifo, E. M. (2020). "Multiple instance learning for sequence data with across bag dependencies". International journal of machine learning and cybernetics, Vol. 11, No. 3, pp. 629-642.
17. Alpaydın, E., Cheplygina, V., Loog, M. and Tax, D. M. (2015). "Single-vs-multipleinstance classification". Pattern recognition, Vol. 48, No. 9, pp. 2831-2838.

18. Tomar, D. and Agarwal, S. (2017). "Multiple Instance Learning Based on Twin Support Vector Machine", in Advances in Computer and Computational Sciences, pp. 497-507, Springer, Singapore.

19. Carbonneau, M. A., Cheplygina, V., Granger, E. and Gagnon, G. (2018). "Multiple instance learning: A survey of problem characteristics and applications". Pattern Recognition, Vol. 77, pp. 329-353.

20. Costa, E. B., Fonseca, B., Santana, M. A., et al. (2017). "Evaluating the effectiveness of educational data mining techniques for early prediction of students' academic failure in introductory programming courses". Computers in Human Behavior, Vol. 73, pp. 247-256.

21. Tharwat, A. (2018). "Classification assessment methods". Applied Computing and Informatics.

22. Fu, G. H., Yi, L. Z. and Pan, J. (2019). "Tuning model parameters in classimbalanced learning with precision-recall curve". Biometrical Journal, Vol. 61, No. 3, pp. 652-664.

23. Thirunavukkarasu, K., Singh, A. S., Rai, P. and Gupta, S. (December 2018). "Classification of IRIS Dataset using Classification Based KNN Algorithm in Supervised Learning", in IEEE 2018 4th International Conference on Computing Communication and Automation (ICCCA), pp. 1-4.

24. Dai, H. J., Wang, F. D., Chen, C. W., et al. (2020). "Cohort selection for clinical trials using multiple instance learning". Journal of biomedical informatics, Vol. 107, pp. 103438. 
25. Dong, L. (February 2006). "A comparison of multi-instance learning algorithms". M.S. thesis, Department of Computer Science, The University of Waikato, Hamilton, New Zealand.

26. Frank, E., Xu, X. (2003). “Applying propositional learning algorithms to multiinstance data". Tech. rep., Department of Computer Science, University of Waikato.

27. Lindig-Leon, C. and Bougrain, L. (October 2015). "A multi-label classification method for detection of combined motor imageries", in 2015 IEEE International Conference on Systems, Man, and Cybernetics, pp. 3128-3133.

28. Guan, X., Liang, J., Qian, Y. and Pang, J., (2017). "A multi-view OVA model based on decision tree for multi-classification tasks". Knowledge-Based Systems, Vol. 138, pp. 208-219. 